\title{
NNLO QCD fits to extract PDFs from HERA inclusive and jet data
}

\author{
Amanda Cooper-Sarkar ${ }^{* \dagger}$ \\ University of Oxford \\ E-mail: amanda.cooper-sarkar@physics.ox.ac.uk
}

The HERAPDF2.0 family, introduced in 2015, is completed with fits HERAPDF2.0Jets NNLO (prel.) based on inclusive HERA data and selected jet production data. The result of a fit with the strong coupling constant, $\alpha_{s}\left(M_{Z}^{2}\right)$, free is $\alpha_{s}\left(M_{Z}^{2}\right)=0.1150 \pm 0.0008(\exp )_{-0.0005}^{+0.0002}$ (model/parameterisation) \pm 0.0006 (hadronisation) \pm 0.0027 (scale). Sets of parton density functions, PDFs, from fits with fixed $\alpha_{s}\left(M_{Z}^{2}\right)=0.115$ and $\alpha_{s}\left(M_{Z}^{2}\right)=0.118$ are presented and compared

European Physical Society Conference on High Energy Physics - EPS-HEP2019 -

10-17 July, 2019

Ghent, Belgium

\footnotetext{
*Speaker.

${ }^{\dagger}$ On behalf of $\mathrm{H} 1$ and ZEUS Collaborations
} 


\section{Introduction}

Deep inelastic scattering (DIS) of electrons on protons, ep, at centre-of-mass energies of up to $\sqrt{s} \approx 320 \mathrm{GeV}$ at HERA has been central to the exploration of proton structure and quark-gluon dynamics as described by perturbative Quantum Chromo Dynamics (pQCD). The combination of $\mathrm{H} 1$ and ZEUS data on inclusive $e p$ scattering and the subsequent pQCD analysis, introducing the family of parton density functions (PDFs) known as HERAPDF2.0[1], was a milestone for the exploitation of the HERA data. The preliminary work presented here represents a completion of the HERAPDF2.0 family with a fit at NNLO to HERA inclusive and jet production data published separately by the ZEUS and H1 collaborations. This was not possible at the time of the original introduction of HERAPDF2.0 because a treatment at NNLO of jet production in $e p$ scattering was not available then.

\section{Procedure and Data}

The name HERAPDF stands for a PQCD analysis within the DGLAP formalism, where predictions from $\mathrm{pQCD}$ are fitted to data. These predictions are obtained by solving the DGLAP evolution equations at LO, NLO and NNLO in the $\overline{\mathrm{MS}}$ scheme. The inclusive and dijet production data which were already used for HERAPDF2.0Jets NLO were again used for the analysis presented here. A new data set [2] published by the H1 collaboration on jet production in low $Q^{2}$ events, where $Q^{2}$ is the four-momentum-transfer squared, was added as input to the fits.

The fits presented here were done in the same way as for all other members of the HERAPDF2.0 family, for full details see [3] and references therein. The fits were performed using the programme QCDNUM within the xFitter framework. Only cross sections for $Q^{2}$ starting at $Q_{\min }^{2}=3.5 \mathrm{GeV}^{2}$ were used in the analysis. All parameter setting were the same as for the HERAPDF2.0Jets NLO fit. The analysis of uncertainties was also performed in exactly the same way.

There were some modifications with respect to the analysis at NLO. They were driven by the usage of the newly available treatment of jet production at NNLO. The jet data were included in the fits at NNLO by calculating predictions for the jet cross sections within the Applfast framework using the NNLO extension of NLOjet++ interfaced to FastNLO in order to achieve the speed necessary for iterative PDF fits. The predictions were multiplied by corrections for hadronisation and $Z^{0}$ exchange before they were used in the fits. A running electro-magnetic $\alpha$ as implemented in the 2012 version of the programme EPRC was used for the treatment of the jet cross sections.

The new treatment of inclusive jet and dijet production at NNLO was only applicable to a slightly reduced phase space compared to HERAPDF2.0Jets NLO. All data points with $\sqrt{\left\langle p_{T}^{2}\right\rangle+Q^{2}} \leq$ $13.5 \mathrm{GeV}$ were excluded, where $p_{T}$ is the transverse energy of the jets. In addition, six data points, the lowest $\left\langle p_{T}\right\rangle$ bin for each $Q^{2}$ region, were excluded from the ZEUS dijet data set because the NNLO predictions for these points were deemed unreliable. In addition, the trijet data which were used as input to HERAPDF2.0Jets NLO had to be excluded as their treatment at NNLO was not available.

The choice of scales was also adjusted for the NNLO analysis. At NLO, the factorisation scale was chosen as $\mu_{\mathrm{f}}^{2}=Q^{2}$, while the renormalisation scale was linked to the transverse momenta, $p_{T}$, of the jets by $\mu_{\mathrm{r}}^{2}=\left(Q^{2}+p_{T}^{2}\right) / 2$. For the NNLO analysis, $\mu_{\mathrm{f}}^{2}=\mu_{\mathrm{r}}^{2}=Q^{2}+p_{T}^{2}$ was chosen. 


\section{Determination of the strong coupling constant}

Jet production data are essential for the determination of the strong coupling constant, $\alpha_{s}\left(M_{Z}^{2}\right)$. In pQCD fits to inclusive DIS data alone, the gluon PDF is determined via the DGLAP equations only, using the observed scaling violations. This results in a strong correlation between the shape of the gluon distribution and the value of $\alpha_{s}\left(M_{Z}^{2}\right)$. Data on jet production cross sections provide an independent constraint on the gluon distribution. Jet and dijet production are also directly sensitive to $\alpha_{s}\left(M_{Z}^{2}\right)$ and thus such data allow for an accurate simultaneous determination of $\alpha_{s}\left(M_{Z}^{2}\right)$ and the gluon distribution.

The HERAPDF2.0Jets NNLO (prel.) fit with free $\alpha_{s}\left(M_{Z}^{2}\right)$ gave a value of

$$
\begin{array}{r}
\alpha_{S}\left(M_{Z}^{2}\right)=0.1150 \pm 0.0008(\exp )_{-0.0005}^{+0.0002}(\text { model } / \text { parameterisation }) \\
\pm 0.0006(\text { hadronisation }) \pm 0.0027 \text { (scale })
\end{array}
$$

This result on $\alpha_{s}\left(M_{Z}^{2}\right)$ is compatible with the world average [4] and it is competitive with other determinations at NNLO.

The HERAPDF2.0Jets NNLO (prel.) fit with free $\alpha_{s}\left(M_{Z}^{2}\right)$ uses 1343 data points and has a $\chi^{2} /$ d.o.f. $=1599 / 1328=1.203$. This can be compared to the $\chi^{2} /$ d.o.f. $=1363 / 1131=1.205$ for HERAPDF2.0 NNLO based on inclusive data only. The similarity of the $\chi^{2} /$ d.o.f. values indicates that the data on jet production do not introduce any tension.

The experimental uncertainty was determined from the fit. The $\chi^{2}$ scan in $\alpha_{s}\left(M_{Z}^{2}\right)$ shown in Fig. 1a) confirmed the value of $\alpha_{s}\left(M_{Z}^{2}\right)$ and the experimental uncertainty. In addition to this the HERAPDF procedure considers model and parameterisation uncertainties and, for jet data, hadronisation uncertainties are also considered, see [1] for details. These additional uncertainties are also shown in Fig. 1a).

A strong motivation to determine $\alpha_{s}\left(M_{Z}^{2}\right)$ at NNLO was the hope to substantially reduce scale uncertainties. This uncertainty was evaluated by varying the renormalisation and factorisation scales by a factor of two, both separately and simultaneously, and taking the maximal positive and negative deviations. The uncertainties were assumed to be $50 \%$ correlated and $50 \%$ uncorrelated between bins and data sets. The result is also shown in Fig. 1a). The scale uncertainty still dominates the uncertainties.

As the input data were changed for the NNLO analysis and the choice of scales were changed with respect to the NLO analysis, a detailed comparison of scale uncertainties will be published after the appropriate reanalysis of the data at NLO. However, the present scale uncertainty, of \pm 0.0027 for the NNLO analysis, is significantly lower than the $+0.0037,-0.0030$ previously observed for the HERAPDF2.0Jets NLO analysis. If the NNLO determination of $\alpha_{s}\left(M_{Z}^{2}\right)$ is performed with the old choice of scales, the value of $\alpha_{s}\left(M_{Z}^{2}\right)$ is further reduced to 0.1135 , well within scale uncertainties.

The question whether data with relatively low $Q^{2}$ bias the determination of $\alpha_{s}\left(M_{Z}^{2}\right)$ arises in the context of the HERA data anlysis for which low $Q^{2}$ is also low $x$. A treatment beyond DGLAP may be necessary because of higher-tiwst terms, $\ln (1 / x)$ terms or even parton saturation. To check for such bias scans were performed with $Q_{\min }^{2}$ set to $3.5 \mathrm{GeV}^{2}, 10 \mathrm{GeV}^{2}$ and $20 \mathrm{GeV}^{2}$ for the inclusive data. The value of $\alpha_{s}\left(M_{Z}^{2}\right)$ at the minumum did not differ significantly with the value of this cut, see [3] for further details. 


\section{H1 and ZEUS preliminary}

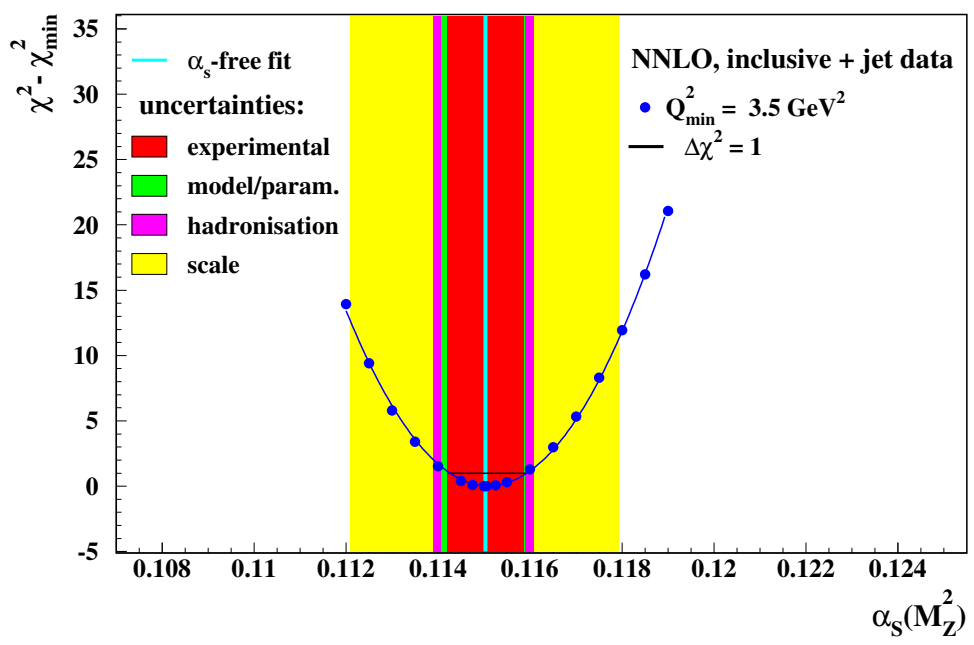

Figure 1: $\Delta \chi^{2}=\chi^{2}-\chi_{\min }^{2}$ vs. $\alpha_{s}\left(M_{Z}^{2}\right)$ for HERAPDF2.0Jets NNLO (prel.) fits with fixed $\alpha_{s}\left(M_{Z}^{2}\right)$ with the standard $Q_{\min }^{2}$ of $3.5 \mathrm{GeV}^{2}$.

\section{The PDFs of HERAPDF2.0Jets NNLO (prel.)}

The PDFs resulting from the HERAPDF2.0Jets NNLO (prel.) fit with fixed $\alpha_{s}\left(M_{Z}^{2}\right)=0.115$ and $\alpha_{s}\left(M_{Z}^{2}\right)=0.118$ are shown and compared in in Fig. 2 at a scale of $Q^{2}=10 \mathrm{GeV}^{2}$. Here, total uncertainties are shown, including experimental, model and parameterisation uncertainties as well as additional hadronisation uncertainties on the jet data. The former value of $\alpha_{S}\left(M_{Z}^{2}\right)$ is chosen because it is the preferred value of these data and the latter value is chosen because it is the PDG value, and because it allows direct comparison to the published PDFs of HERAPDF2.0 NNLO based on inclusive data only. These PDFs are very similar, see ref [3], indicating that the jet data do not change PDF shapes for fixed $\alpha_{s}\left(M_{Z}^{2}\right)$, but they have impact on the extracted value of $\alpha_{s}\left(M_{Z}^{2}\right)$, when it is allowed to be free. The comparison of the new HERAPDF2.0Jets NNLO (prel.) fits with differing values of $\alpha_{s}\left(M_{Z}^{2}\right)$, Fig. 2,shows a significant difference in the gluon distributions, as expected given the correlation between the gluon PDF shape and the value of $\alpha_{s}\left(M_{Z}^{2}\right)$.

The quality of the fit to the jet data is illustrated in Figs. 3, 4. Further data/fit comparisons may be found in ref. [3].

\section{Summary}

The HERA data set on inclusive ep scattering as introduced by the ZEUS and H1 collaborations, together with selected data on jet production, published separately by the two collaborations, were used as input to NNLO fits called HERAPDF2.0Jets NNLO (prel.). They complete the HERAPDF2.0 family. A fit with free $\alpha_{s}\left(M_{Z}^{2}\right)$ gave $\alpha_{s}\left(M_{Z}^{2}\right)=0.1150 \pm 0.0008(\exp )_{-0.0005}^{+0.0002}(\mathrm{mo}-$ $\mathrm{del} /$ parameterisation) \pm 0.0006 (hadronisation) \pm 0.0027 (scale). A preliminary set of PDFs with a full analysis of uncertainties was obtained from a HERAPDF2.0Jets NNLO (prel.) fit with fixed $\alpha_{s}\left(M_{Z}^{2}\right)=0.115$. These PDFs were compared to PDFs from a similar fit with fixed $\alpha_{s}\left(M_{Z}^{2}\right)=0.118$ 

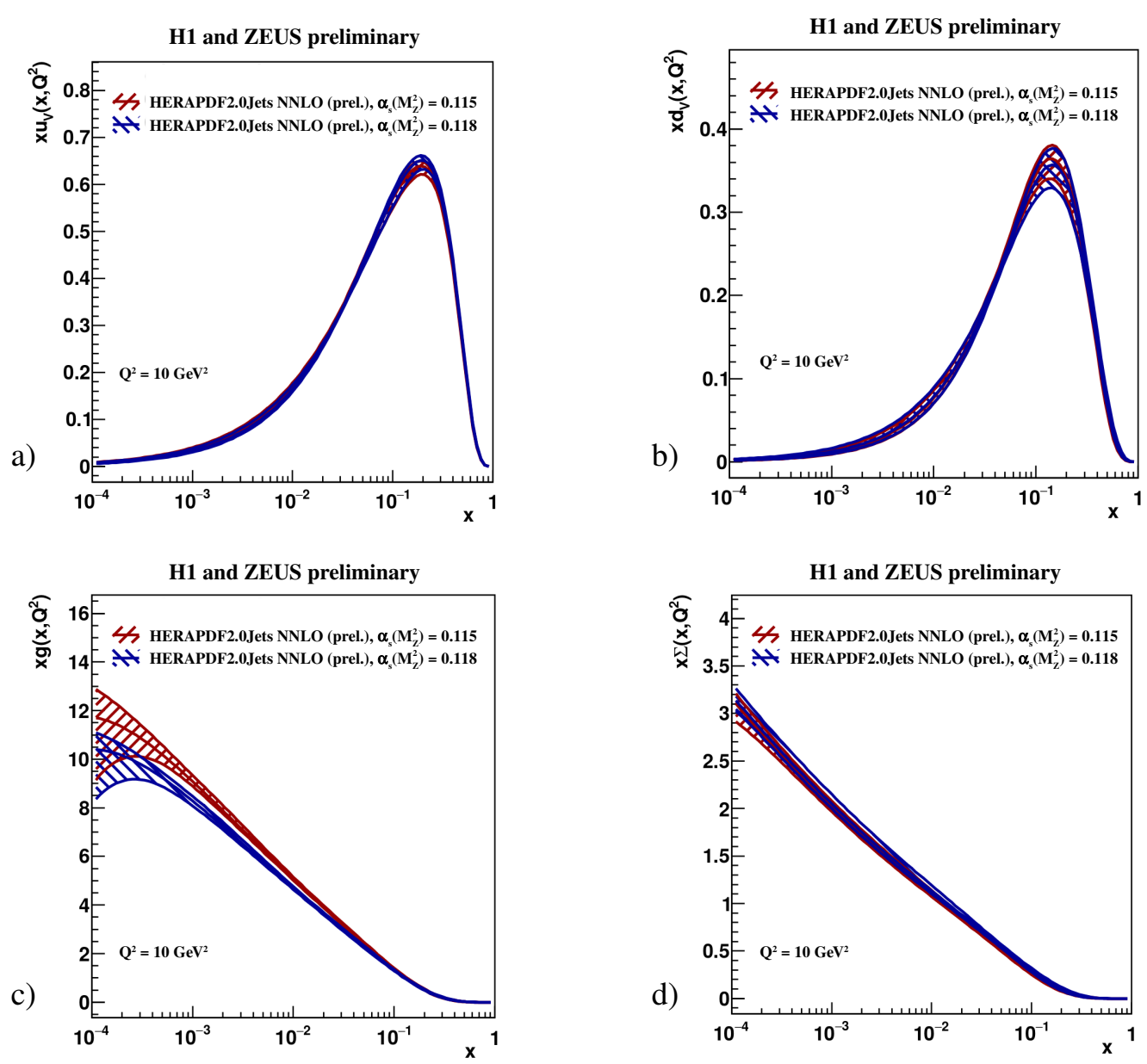

Figure 2: Comparison of the parton distribution functions a) $x u_{v}$, b) $x d_{v}$, c) $x g$ and d) $x \Sigma=x(\bar{U}+\bar{D})$ of HERAPDF2.0Jets NNLO (prel.) with fixed $\alpha_{s}\left(M_{Z}^{2}\right)=0.115$ and $\alpha_{s}\left(M_{Z}^{2}\right)=0.118$ at the scale $Q^{2}=10 \mathrm{GeV}^{2}$. The total uncertainties are shown as differently hatched bands.

and the PDFs from HERAPDF2.0 NNLO based on inclusive data only. All these PDFs are very similar.

\section{References}

[1] H. Abramowicz et al. [ZEUS and H1 Collaboration], Eur. Phys. J. C 75, 580 (2015), [arXiV:1506.06042].

[2] V. Andreev et al. [H1 Collaboration], Eur. Phys. J. C 77, 215 (2017), [arXiV:1611.03421].

[3] H1prelim-19-041, ZEUS-prel-19-001, www-h1.desy.de/publications/htmlsplit/H1 prelim-19-041.long.html, see also arXiV:1906.01884

[4] M. Tanabashi et al. [Particle Data Group], Phys. Rev.D 98, 030001 (2018) 


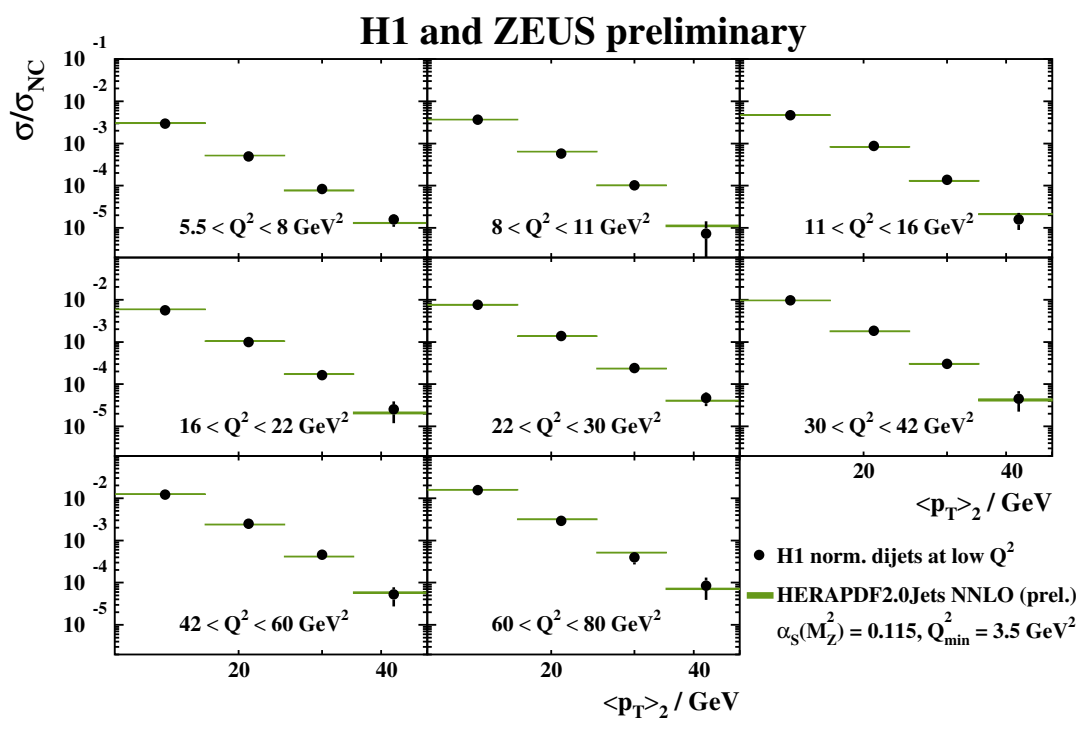

Figure 3: The predictions of HERAPDF2.0Jets NNLO (prel.) with fixed $\alpha_{s}\left(M_{Z}^{2}\right)=0.115$ compared to H1 normalised dijet data at low $Q^{2}$

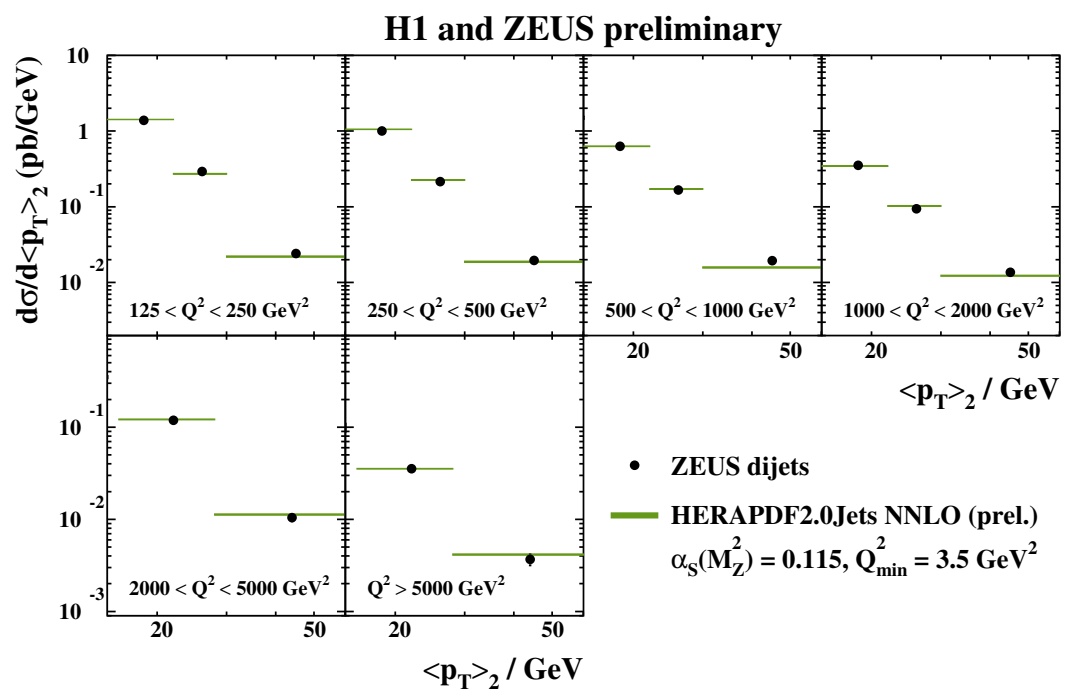

Figure 4: The predictions of HERAPDF2.0Jets NNLO (prel.) with fixed $\alpha_{s}\left(M_{Z}^{2}\right)=0.115$ compared to ZEUS dijet data at high $Q^{2}$ 